\title{
Depósitos del Aptiano inferior con amonitas y rudistas, Punta Espada, Alta Guajira (Uribia - Colombia). Litoestratigrafía y apreciaciones regionales
}

\begin{abstract}
Pedro Patarroyo $^{1^{*}}$ (D) ; †Stephan Götz ${ }^{2}$
Forma de citar: Patarroyo, P.; Götz, S. (2020). Depósitos del Aptiano inferior con amonitas y rudistas, Punta Espada, Alta Guajira (Uribia - Colombia). Litoestratigrafía y apreciaciones regionales. Boletín de Geología, 42(3), 227-241. https://doi.org/10.18273/revbol.v42n3-2020010
\end{abstract}

\begin{abstract}
Resumen
En depósitos del valle Piedra del Destino (Punta Espada, Uribia - La Guajira, Colombia) se presentan biomicritas, bioesparitas y lodolitas calcáreas, en las que se encontraron Dufrenoyia sp., D. cf. hansbuergli, Cheloniceras sp. Pseudohaploceras cf. liptoviense y Amphitriscoelus waringi con un rango estratigráfico del Aptiano inferior. La literatura refiere que dichos depósitos hacen parte de la Formación Cogollo, pero con base en el análisis de la litoestratigrafía, la fauna fósil, la tectónica y la continuidad de los cuerpos de roca, difícilmente se recomienda aplicar la nomenclatura litoestratigráfica del occidente de Venezuela para la Alta Guajira, región que involucra los depósitos cretácicos de Punta Espada. La fauna encontrada muestra influencia tanto del mar del Tetis como del entorno regional por provincialismo.
\end{abstract}

Palabras clave: Depósitos del Aptiano; Amonitas; Rudistas; Alta Guajira; Colombia.

\section{Lower Aptian deposits with ammonites and rudists, Punta Espada, Alta Guajira (Uribia - Colombia). Lithostratigraphy and regional commentaries}

\begin{abstract}
In the Piedra del Destino valley (Punta Espada, Uribia - La Guajira, Colombia) there are biomicrites, biosparites and calcareous shales that contain Dufrenoyia sp., D. cf. hansbuergli, Cheloniceras sp. Pseudohaploceras cf. liptoviense and Amphitriscoelus waringi with a lower Aptian range. The bibliography relate that those deposits are part of the Cogollo Formation, but taken into account the lithostratigraphical analysis, fossil fauna, tectonic and body rock continuity, it is not recommendable to apply the lithostratigraphical nomenclature from western Venezuela to the Alta Guajira region where are evolved the Punta Espada Cretaceous deposits. The collected fossils show both influence of the Tethys domain and as regional provincialism.
\end{abstract}

Keywords: Aptian deposits; Ammonites; Rudists; Alta Guajira; Colombia.

\footnotetext{
'Departamento de Geociencias, Universidad Nacional de Colombia, Bogotá, Colombia. (*) pcpatarroyog@unal.edu.co ${ }^{2} \uparrow(1964$ - 2012). Heidelberg Universität, Heidelberg, Germany.
} 


\section{Introducción}

La sucesión sedimentaria del Cretácico del norte continental de Colombia ha venido siendo investigado desde mediados del siglo XX, pero el conocimiento estratigráfico sobre las unidades litoestratigráficas asociadas desafortunadamente es escaso. En el sector de la Alta Guajira (Colombia) hay una sucesión del Cretácico Inferior que involucra diferentes unidades litoestratigráficas, con variaciones composicionales y granulométricas, que reflejan diferentes ambientes de depósito y que en algunos casos alberga fauna fósil. Las publicaciones relacionadas con la estratigrafía y paleontología del Cretácico de la península de La Guajira son pocas y desafortunadamente no tan detalladas, debido posiblemente a las dificultades de acceso a este sector de Colombia. Renz $(1956,1960)$, Bürgl (1960) y Rollins $(1960,1965)$ aportaron valiosos datos estratigráficos para su momento, discutiendo aspectos sobre la litoestratigrafía y la paleontología, pero no presentando ilustraciones de los fósiles. Zuluaga et al. (2009) solo sintetizaron la estratigrafía cretácea existente en una cartografía geológica y Salazar (2010) con su tesis de maestría describió la Formación Palanz, que involucra depósitos continentales y transicionales.

Con base en amonitas, corales, foraminíferos, rudistas y otros bivalvos Renz (1956, 1960), Bürgl (1960) y
Rollins (1960, 1965) reconocieron la existencia de depósitos calcáreos del Aptiano en la Alta Guajira.

Teniendo en cuenta el reconocimiento directo de la sucesión del Cretácico Inferior en la Alta Guajira (Colombia), se logran diferenciar unidades litoestratigráficas, a partir de lo cual se discuten planteamientos sobre la nomenclatura estratigráfica, se presentan sus afloramientos, se ilustran los fósiles recolectados, se indica su posición estratigráfica y su sistemática.

La asociación fósil del Aptiano encontrada en la Alta Guajira y mostrada en esta contribución, presenta similitud con asociaciones de sucesiones del Aptiano reportadas en México, Texas, Venezuela y en general en otros lugares del Caribe, lo cual ayuda a entender relaciones paleobiogeográficas regionales e interregionales.

\section{Localización}

El área de trabajo se localiza cerca al asentamiento de Punta Espada, ubicado en Uribia (La GuajiraColombia) en la península de La Guajira (Figura 1). Esta zona árida se conoce como Alta Guajira, que se ubica al NE cerca del límite con Venezuela.

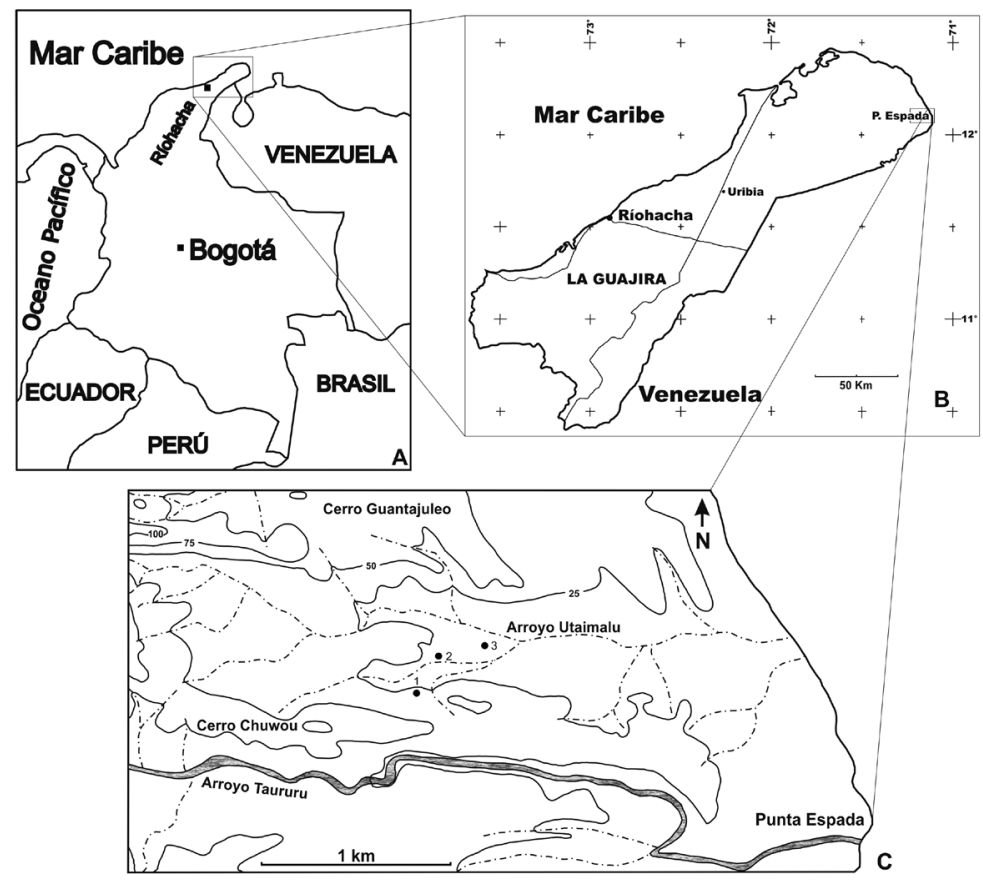

Figura 1. Localización del área de estudio. A. Ubicación de La Guajira en el norte de Colombia. B. Departamento de La Guajira con la ubicación del casco urbano de Uribia y del caserío de Punta Espada. C. Sector de Punta Espada en donde aparece el valle de Piedra del Destino con los lugares de colecta. 1 tramo estratigráfico más bajo, 2 intermedio y 3 más alto. 
Inmediatamente al NE del asentamiento de Punta Espada se ubica el valle Piedra del Destino, en el cual, se encuentran los depósitos aquí investigados, y por donde en época de lluvias discurren las aguas por el arroyo Utaimalu (Figura 1).

\section{Marco Teórico}

La geología del norte continental de Colombia es muy especial, debido a que, sobre los bloques de este sector, la placa del Caribe ejerció una gran influencia tectónica. Por lo tanto, es posible identificar allí las fallas de Oca, Cuisa o Cuiza y Huimatirra (cf. Renz, 1960; Rollins, 1960, 1965; MacDonald, 1964, 1965; Álvarez, 1967; Irving, 1971), que son lineamientos principales del área de la Media y Alta Guajira. Las fallas de Oca y de Cuisa (Figura 2) se consideran fallas de rumbo y tienen continuidad hacia Venezuela, aunque Gómez (2001) indica una mayor complejidad en relación con la historia del movimiento a lo largo del plano de la Falla de Cuisa. Al sur y al norte de la Falla de Cuisa es posible encontrar depósitos cretácicos, cuyos cuerpos de roca pueden recibir las mismas denominaciones. En el bloque norte de la Falla de Cuisa, los depósitos del Cretácico se reconocen dentro de las formaciones Palanz, Yuruma, "Cogollo", "Maraca", "La Luna", Guaralamai y Parauinkrein (Parabanclen) teniendo en cuenta a Renz (1960), Bürgl (1960), Rollins (1960, 1965), Zuluaga et al. (2009) y Salazar (2010). Sobre estas sedimentitas se ubican unidades del Cenozoico incluyendo depósitos eólicos recientes (ver esquema geológico Figura 2).

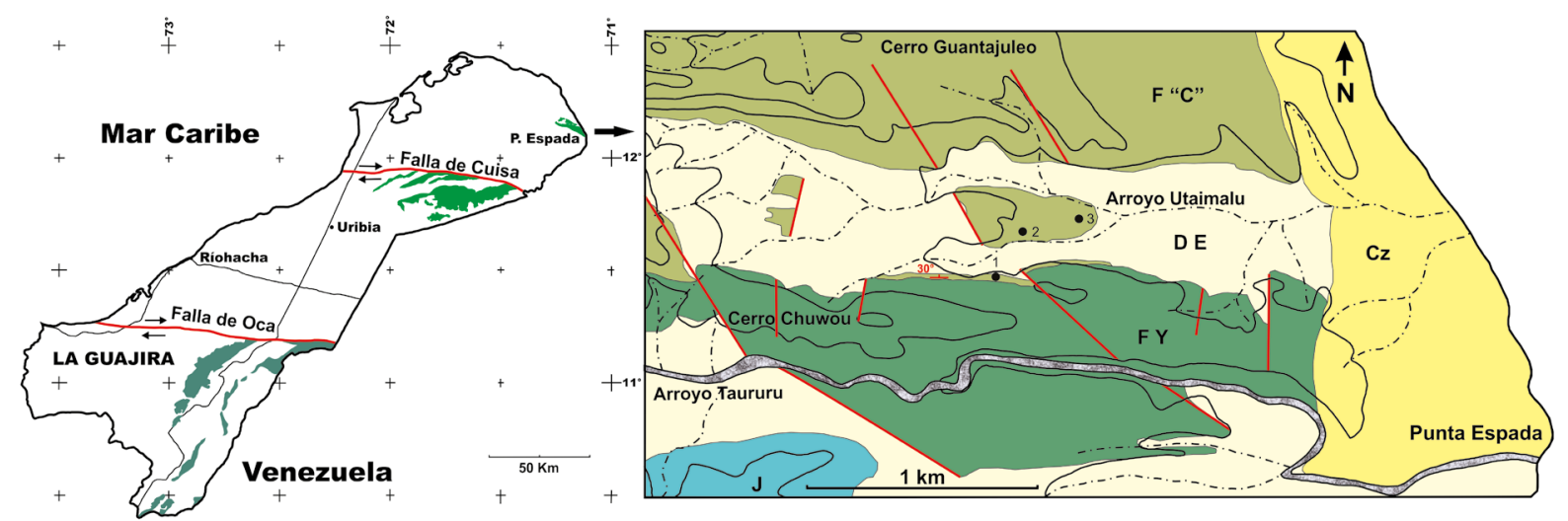

Figura 2. Mapa del departamento de La Guajira (izquierda) en donde aparecen los contornos que ocupan las sucesiones sedimentarias del Cretácico al norte y al sur de la Falla de Oca con base en Mercado (1999). En relación con la Falla de Oca, al norte aparecen las unidades del Cretácico asociadas a la litoestratigrafía de la Alta Guajira y al sur se localizan las unidades litoestratigráficas de la serranía del Perijá. Esquema geológico del área de Punta Espada con los sitios de colecta (derecha), con base en fotogeología, observaciones directas de campo y en Salazar (2010). J - Depósitos sedimentarios del Jurásico, F Y - Formación Yuruma, F “C” - Formación "Cogollo”, Cz - Depósitos del Cenozoico, D E - Depósitos eólicos. Líneas rojas Lineamientos o fallas y dato de rumbo y buzamiento.

En el sector investigado Renz (1960), Bürgl (1960) y Rollins (1960, 1965) realizaron las mejores propuestas cartográficas, mientras que Zuluaga et al. (2009) increíblemente ignoraron dicha cartografía y desconocieron los diferentes planteamientos litoestratigráficos.

\section{Consideraciones sobre la estratigrafía y contexto regional}

En este apartado sobre estratigrafía se aborda la situación de las unidades litoestratigráficas inferior, superior y en específico de la que contiene los depósitos del Aptiano de la Alta Guajira y sus fósiles. Con base en lo cual se plantea un escenario regional, algo diferente al que la mayoría de investigadores consideraba.

\section{El término Cogollo}

Garner (1926, p. 679) introdujo la denominación "Rio Cogollo limestone" en el oeste de Venezuela (serranía del Perijá) al sur oeste de la Alta Guajira, y de forma muy general "Type locality: forms hills and is exposed in Cogollo River, northwestern part of District of Perija, Zulia. Massive, grey, crystalline, fossiliferous limestone". Este término ha tenido diferentes aplicaciones y límites, ya sea como formación o como grupo, por lo tanto, hay confusión y existen erróneas y falsas interpretaciones estratigráficas.

Lo cual significa que la unidad litoestratigráfica Cogollo tiene diferentes interpretaciones tanto en Colombia como en Venezuela. La unidad litoestratigráfica Cogollo, en Venezuela, incluye una sucesión entre el 
límite superior de la Formación Río Negro y el límite inferior de la Formación La Luna. Allí, lo que se conoce como Grupo Cogollo se subdivide en las formaciones Apón, Lisure y Maraca (Rod y Maync, 1954). Su rango estratigráfico puede estar comprendido entre Aptiano y Cenomaniano, pero es posible que involucre depósitos del Barremiano en su parte inferior (Renz, 1959).

En el Perijá del lado colombiano y sectores aledaños se han usado otras nomenclaturas para la sucesión entre la Formación Río Negro y la Formación La Luna como lo son Formación Lagunita, Formación Aguas Blancas, Miembro Ánimas y Miembro Tocuy, Grupo Cogollo, Miembro Maracas (cf. Durham, 1946; Govea y Dueñas, 1975; Cáceres et al., 1980; Hernández, 2003).

Al sur del Perijá, para el sector del Catatumbo (Concesión Barco) en Colombia, la situación es igualmente confusa, Miller en Richards (1968) divide el Grupo Cogollo en las formaciones Apón, Lisure y Maraca, para la sucesión que se encuentra entre la Formación Río Negro y la Formación La Luna. Además, Richards (1968, p. 2330), para esta misma sucesión, usa los términos Formación Apón, Formación Aguardiente y Formación Capacho, indicando "The Capacho Formation in the Barco area was mistakenly called Cogollo Formation by Notestein et al. (1944, p. 1178-82)".

Otto Renz, con base en su conocimiento de las unidades litoestratigráficas del occidente de Venezuela, lleva a la Alta Guajira en Colombia parte de la nomenclatura litoestratigráfica de este sector de Venezuela. Para la península de La Guajira, Renz (1956) cita primero el término "Cogollo" (Tabla 1) con el rango de formación, pero luego Renz (1960) usa para esta área la denominación "Cogollo" pero con rango de grupo (Tabla 1). Rollins (1960, 1965) mantiene la denominación Grupo "Cogollo" para una sucesión entre las formaciones Yuruma superior (Barremiano) y "La Luna" (Turoniano - Coniaciano), pero subdivide este grupo (Tabla 1) en la Formación "Cogollo" inferior y la Formación "Maraca" (cf. Patarroyo, 2011; Patarroyo y Götz, 2013, 2014). Rollins (1960, 1965) modifica el límite inferior de la Formación "Cogollo" inferior (Tabla 1), lo cual implica que excluyó la parte inferior del "Cogollo" de Renz $(1956,1960)$ y que a su vez adicionó al techo de la Formación Yuruma superior (Tabla 1). Este nivel excluido de "Cogollo" corresponde a la "unidad inferior formada predominantemente de calizas densas gris oscuras" de Renz (1960, p. 333). Por otro lado, Zuluaga et al. (2009) en la zona de Punta Espada, utilizaron denominaciones informales para su cartografía geológica como K5 para las formaciones Yuruma y "Cogollo" inferior y K6 para la Formación "Maraca". Desafortunadamente y como se hace evidente no existe un uso con criterios unificados de la litoestratigrafía para la sucesión del Cretáceo en el área de la Alta Guajira. Tanto así que Zuluaga et al. (2009) indican que no hay un límite claro entre la Formación Yuruma y la unidad informal K5 (que incluye depósitos de la Formación Yuruma), porque la sucesión litológica es muy similar y solo con la bioestratigrafía puede ser posible encontrar este límite. Al respecto, Bürgl (1960) indicó, que Renz (1956) incluyó depósitos de Barremiano y Aptiano inferior en la Formación Yuruma superior, y depósitos del Aptiano y Cenomaniano superior en la Formación "Cogollo".

Tabla 1. Esquema comparativo de los rangos litoestratigráficos de unidades del Cretácico Inferior y sus límites con base en Renz (1956, 1960) y Rollins (1960, 1965).

\begin{tabular}{|c|c|c|c|c|}
\hline \multirow{5}{*}{ 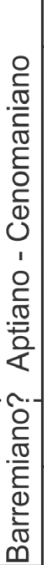 } & Renz (1956) & Renz (1960) & \multicolumn{2}{|r|}{ Rollins $(1960,1965)$} \\
\hline & \multirow{3}{*}{$\begin{array}{l}\text { Formación } \\
\text { "Cogollo" }\end{array}$} & \multirow{3}{*}{ Grupo "Cogollo" } & \multirow{2}{*}{ 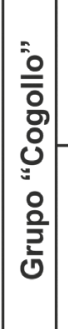 } & Formación "Maraca" \\
\hline & & & & $\begin{array}{c}\text { Formación } \\
\text { "Cogollo" inferior }\end{array}$ \\
\hline & & & \multirow{2}{*}{\multicolumn{2}{|c|}{$\begin{array}{l}\text { Formación } \\
\text { Yuruma superior }\end{array}$}} \\
\hline & $\begin{array}{c}\text { Formación } \\
\text { Yuruma superior }\end{array}$ & Formación Yuruma & & \\
\hline
\end{tabular}


Para la sucesión del Aptiano - Cenomaniano de la Alta Guajira no se puede, hasta ahora, determinar una clara subdivisión litológica como se presenta en Venezuela para el Grupo Cogollo. Además, en la serranía del Perijá, en Colombia y en Venezuela, la Formación Cogollo suprayace a depósitos siliciclásticos transicionales de la Formación Río Negro, mientras que en la Alta Guajira estaría suprayaciendo a depósitos marinos calcáreos de la Formación Yuruma. Si bien MacDonald $(1964,1965)$ afirma que el Grupo "Cogollo", en el área de Punta Espada, descansa sobre depósitos de la Formación Río Negro, que en realidad corresponden a los de la Formación Palanz (cf. Renz, 1960; Salazar, 2010). Igualmente, MacDonald (1964, 1965) indica, que allí no se pueden reconocer tres divisiones litoestratigráficas del Grupo Cogollo como en el Perijá y que la parte arenácea equivalente a la Formación Lisure no se presenta. Por lo tanto, el uso de la denominación Cogollo en la Alta Guajira, al norte de Colombia, no es recomendado debido a la confusión que genera y a las contradicciones litoestratigráficas anteriormente mencionadas. Más aún sin tener en cuenta la posición tectónica y la historia geológica de los bloques que actualmente conforman la zona de la Alta Guajira (cf. Hernández et al., 2003; Ramírez et al., 2015, fig. 33), bloques que se encontraban al occidente de su posición actual.

Para poder solucionar los problemas litoestratigráficos de la Alta Guajira se debe adelantar una descripción detallada de la sucesión (cf. Patarroyo, 2020), con base en lo que se aclararán los límites de las unidades y de paso se determina la distribución vertical de los fósiles.

Para el presente trabajo (ver esquema geológico Figura 2) se sigue la propuesta de Rollins (1960, 1965) en relación con los depósitos del Barremiano - Aptiano, en la que el nivel potente de biomicritas, que Renz $(1956,1960)$ incluía en la parte baja de la Formación "Cogollo", hace parte del intervalo superior de la Formación Yuruma. En campo, dicho nivel litoestratigráfico, presenta geoformas destacadas y a su vez en su parte superior marca un cambio morfológico muy claro, que ayuda a seguirlo lateralmente con fines cartográficos.

Para introducir una nueva denominación a lo que se conoce como Formación "Cogollo" inferior (Rollins, 1960, 1965), se debe buscar en la Alta Guajira, una sección continua o compuesta, de donde se extraerá la denominación, ya sea con un nombre geográfico o ancestral, acompañado de un término litológico representativo.
Así que, las sedimentitas y los fósiles del Aptiano de la presente investigación se ubican en la parte más baja de lo que en el valle Piedra del Destino (área de Punta Espada) se debe considerar como Formación "Cogollo" inferior en el sentido de Rollins (1960, 1965).

\section{Fauna de "Cogollo" en la Alta Guajira}

Renz (1960) para la parte inferior del Grupo "Cogollo" en el área de La Guajira citó Colombiceras aff. codazzianum (Karsten) $(?=$ Dufrenoyia aff. codazziana cf. Bogdanova y Hoedemaeker, 2004), Deshayesites cf. colombianus Riedel (? = Neodeshayesites colombianus, cf. Etayo-Serna, 1979, 1981; Bogdanova y Hoedemaeker, 2004), Parahoplites obliquus Riedel (= Riedelites ubliquum, cf. Etayo-Serna, 1979) y Uhligella sp. del Aptiano. Choffatella decipiens Schlumberger, Exogyra, Trigonia, Idonearca, Pithonella, Globigerina, Trocholina, Textularia, Cuneolina, Vaginulina, Discorbis y equinoideos. Para la parte superior del Grupo "Cogollo" Venezoliceras sp. y Pervinquieria sp. del Albiano.

Bürgl (1960, p. 149) indicó del Aptiano la presencia de Cymatoceras perstriatum (Steuer), Eogaudryceras sp., Valdedorsella inca (Forbes), "Zürcherella zürcheri" (Jacob y Tobler), Uhligella sp., "Deshayesites" aff. codazzianus (Karsten) (? = Dufrenoyia aff. codazziana, cf. Bogdanova y Hoedemaeker, 2004), "Parahoplites" obliquus Riedel (= Riedelites ubliquum, cf. EtayoSerna, 1979), Cheloniceras cf. subnodosocostatum (Sinzow), Colombiceras (?) sp. y rudistas.

Rollins (1960, 1965, p. 36) citó de "Cogollo" inferior "Deshayesites" sp., "D". stutzeri Riedel (? = Neodeshayesites stutzeri (Riedel), Melchiorites? sp., Colombiceras sp., Uhligella sp., Ancyloceras? cf. degenhardtii inflatum (Karsten), Protocardia sp., P. elongata Gerhardt, Lucina porrecta Gerhard, Neaera convergens Gerhardt e Inoceramus sp. Para la Formación "Maraca" Orbitolina concava var. texana Roemer, "Trigonia" (Quadratotrigonia) hondaana Lea, "T." (Notoscabrotrigonia) tocaimaana Lea, Ostrea sp., O. scyphax y Exogyra n. sp. ?. Lo cual indica que la sucesión estratigráfica de lo que se considera como Grupo "Cogollo" en la península de La Guajira abarca entre el Aptiano y el Cenomaniano.

Por otro lado, Zuluaga et al. (2009) afirmaron que los rudistas están en la unidad K6 o Formación "Maraca" con un rango de Albiano, pero contradictoriamente Zuluaga et al. (2009, p. 317) según Rollins (1965) anotaron que, en la parte inferior de la Formación 
"Maraca" se reportan depósitos del Aptiano con base en su contenido fósil.

\section{Metodología}

Se realizo un análisis de los estudios previos publicados sobre la estratigrafía del Cretácico de la Alta Guajira, que se constituyó en el insumo inicial de la investigación aquí presentada.

Para la mitad del año 2009, junto con estudiantes de Geología de la Universidad Nacional de Colombia y geólogos de Ingeominas (hoy Servicio Geológico Colombiano), se visitó la península de La Guajira con el fin de realizar una práctica académica de campo. Allí en corto tiempo, con base en la caracterización litológica y con diferentes hallazgos fósiles, se reconoció la sucesión cretácica. De tal suerte, con posición estratigráfica fue posible recolectar amonitas y rudistas del Aptiano en la misma localidad. Además, en sectores aledaños, se encontraron depósitos y fósiles del Barremiano, Albiano y Cenomaniano (Patarroyo, 2011, 2020).

Durante 2010 y 2011 en la Universidad de Heidelberg (Alemania), Stephan Götz (1964 - 2012), realizó una serie de cortes progresivos con el equipo G\&N MPS 2 - R300 (precision surface grinding), a un bloque calcáreo con ejemplares de rudistas del valle Piedra del Destino al norte del caserío de Punta Espada.

Con el equipo de corte se obtuvieron superficies pulidas, que en este caso se realizaron perpendicularmente al eje mayor del bivalvo, fijando y estabilizando la muestra con material sintético. La máquina se programó para que realizara micrométricamente y de forma progresiva cada corte de alta precisión. A cada corte pulido, paralelos entre sí, se les tomó una fotografía. Así que, de forma ordenada y secuencialmente se captó la progresión de los cortes, con lo cual se logró la reconstrucción volumétrica para un ejemplar. Con base en la sucesión de fotografías para cada uno de los cortes realizados, a partir del programa Analytics and 3D Reconstruction (DICOM, Vector and 3D software) en una estación Mac Pro, se obtuvo la animación y reconstrucción volumétrica digital de los rudistas.

Desafortunadamente, Stephan Götz especialista en este grupo de bivalvos, antes de su fallecimiento, no alcanzó a indicar la especie o el género de los fósiles, pero se conoce que los rudistas presentados en esta contribución son de Amphitriscoelus waringi Harris y Hodson del Aptiano (Mitchell, S.F., comunicación escrita). Así que honrando la memoria de Stephan y su empeño en la investigación sobre los rudistas finalmente se presenta esta contribución.

Los ejemplares de amonitas fueron colectados directamente en el terreno, preparados o limpiados con métodos convencionales a partir del uso de martillos de peña, cinceles, agujas y martillo neumático. Estos fósiles fueron recubiertos con cloruro de amonio para ser fotografiados y luego tratados digitalmente.

\section{Resultados}

\section{Sucesión del Aptiano en Piedra del Destino}

En el camino entre el asentamiento de Punta Espada y Párauinkrein o Parabanclen es posible encontrar una sucesión que incluye depósitos del Barremiano, Aptiano, Albiano, Cenomaniano y Turoniano (Patarroyo, 2011, 2020; Patarroyo y Götz, 2013, 2014).

En el valle Piedra del Destino (Figura 1C, Figura 2, Figura 3), inicialmente se encuentra un banco muy potente de calizas (FY en Figura 3, Figura 4A) de la parte alta de la Formación Yuruma (Figura 3) en el sentido de Rollins (1960, 1965), en donde se reconocen micritas grises y negras, biomicritas en capas gruesas y muy gruesas (Figura 5). Luego, estratigráficamente arriba y a la base de la Formación "Cogollo" (Figura 3, Figura 5) siguiendo a Rollins $(1960,1965)$, continúan intercalaciones de lodolitas calcáreas negras con capas medias a gruesas de micritas y biomicritas (lugar 1 de colecta en Figura 1C y esquema geológico Figura 2, Figura 4B), como indicaron Renz (1960) y Bürgl (1960). Allí la orientación de los estratos es E-W con $30^{\circ} \mathrm{N}$ de inclinación (ver esquema geológico Figura 2). Más arriba estratigráficamente (lugar 2 de colecta en Figura 1C y Figura 2, Figura 3, Figura 5), los macrofósiles bentónicos, que incluyen los rudistas, se pueden encontrar en la biomicritas o bioesparitas, ya sea con distribución aleatoria (Figura 4C, Figura 7C) o en posición de vida (Figura 7A, 7B, 7D, 7E). La fragmentación y la distribución caótica de los organismos es común (Figura 4C, Figura 7C).

Continuando la sucesión estratigráfica, en las lodolitas calcáreas negras (lugar 3 de colecta en Figura 1C y Figura 2, Figura 3, Figura 4D, Figura 5), los fósiles están paralelos a los planos de estratificación, pero deformados (Figura 4E-4F). Se debe aclarar que la deformación ocurrió después de la diagénesis fósil, debido a la deformación volumétrica de las amonitas. 


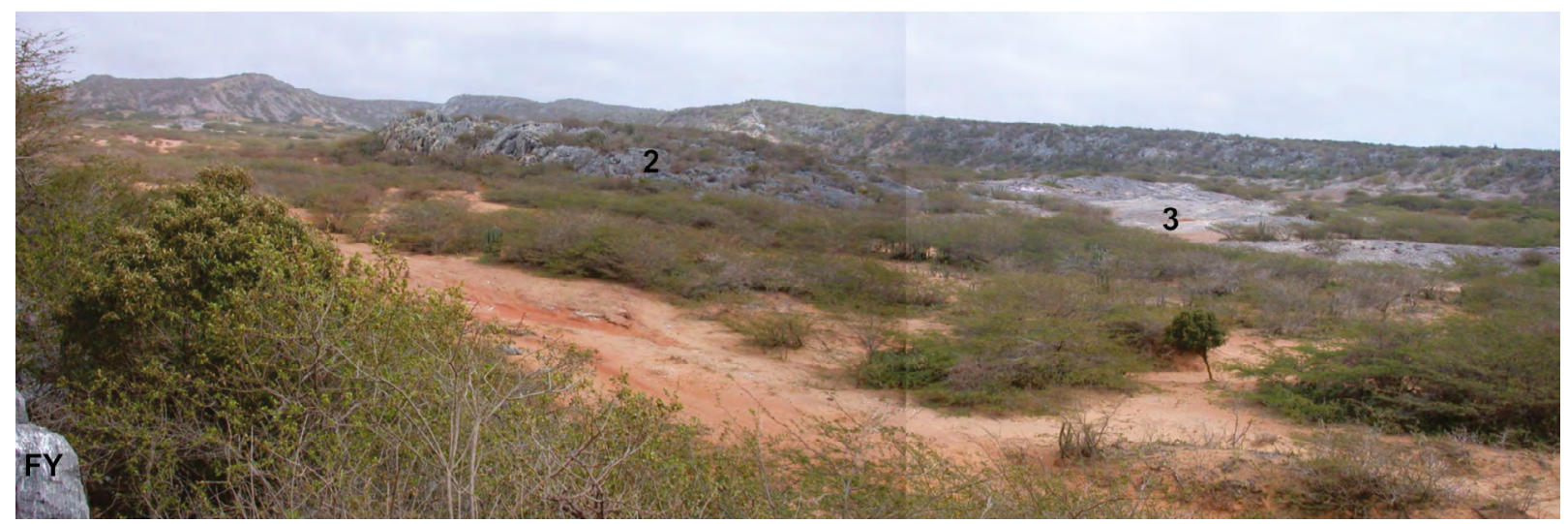

Figura 3. Fotomontaje de la panorámica del valle de Piedra del Destino. FY capas más altas de la Formación Yuruma. 2 y 3 puntos de colecta en relación con la Figura 1C. Fotografías tomadas desde las calizas de la parte alta de la Formación Yuruma (sur), al centro (2) se observa un pequeño montículo con la gran concentración de rudistas. A continuación, el nivel de lodolitas físiles (3).
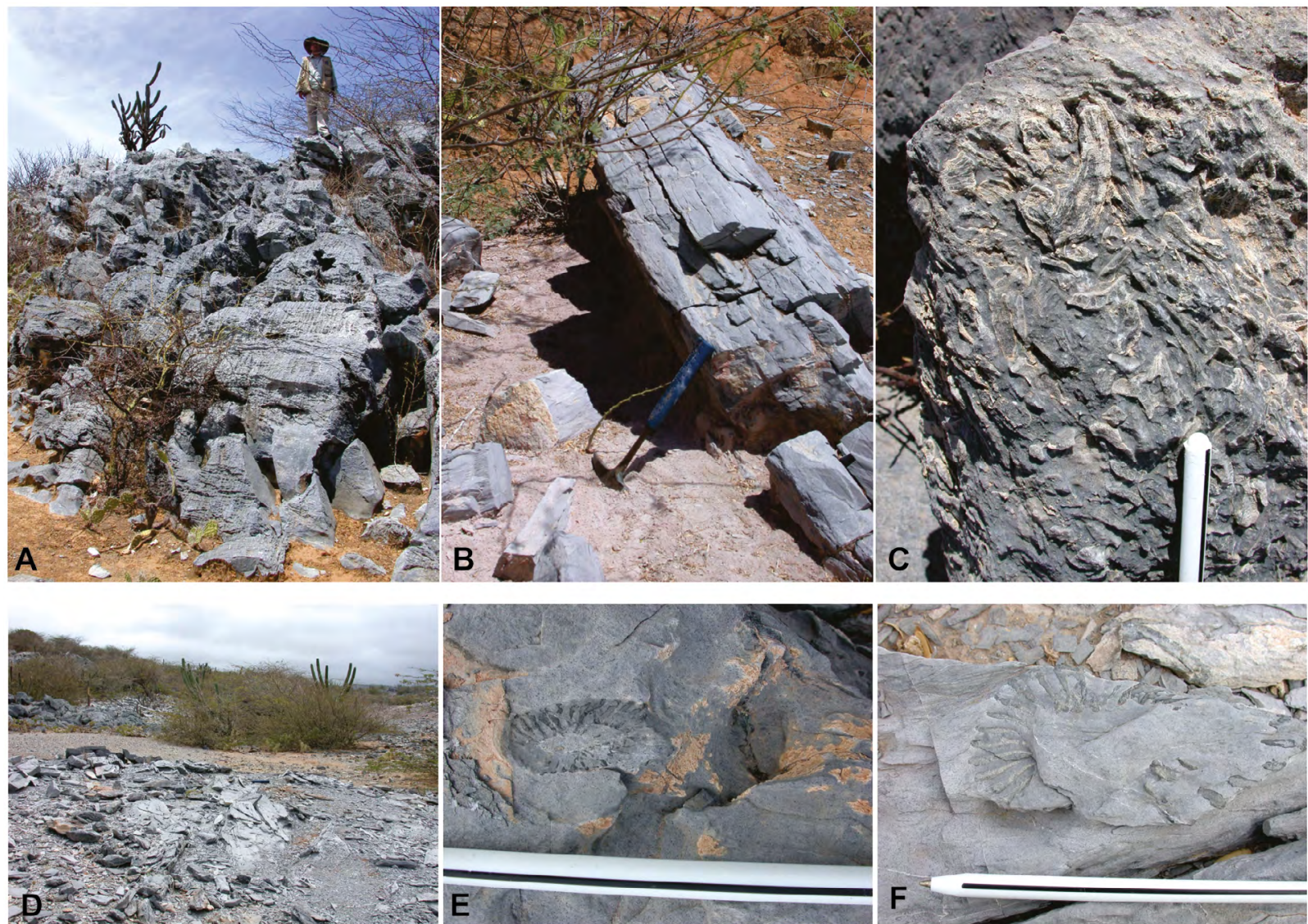

Figura 4. A. Nivel calcáreo potente de la parte alta de la Formación Yuruma. B. Intervalo estratigráfico de la parte baja de la Formación "Cogollo" con lodolitas calcáreas y biomicritas intercaladas (Lugar 1 de colecta Figura 1C). C. Intervalo con rudistas (Lugar 2 de colecta Figura 1C y Figura 2, Figura 3). D-F. Segmento de lodolitas calcáreas (Lugar 3 de colecta Figura 1C y Figura 2, Figura 3). E y F. fósiles deformados del segmento de lodolitas calcáreas (Lugar 3 de colecta Figura 1C y Figura 2, Figura 3). 


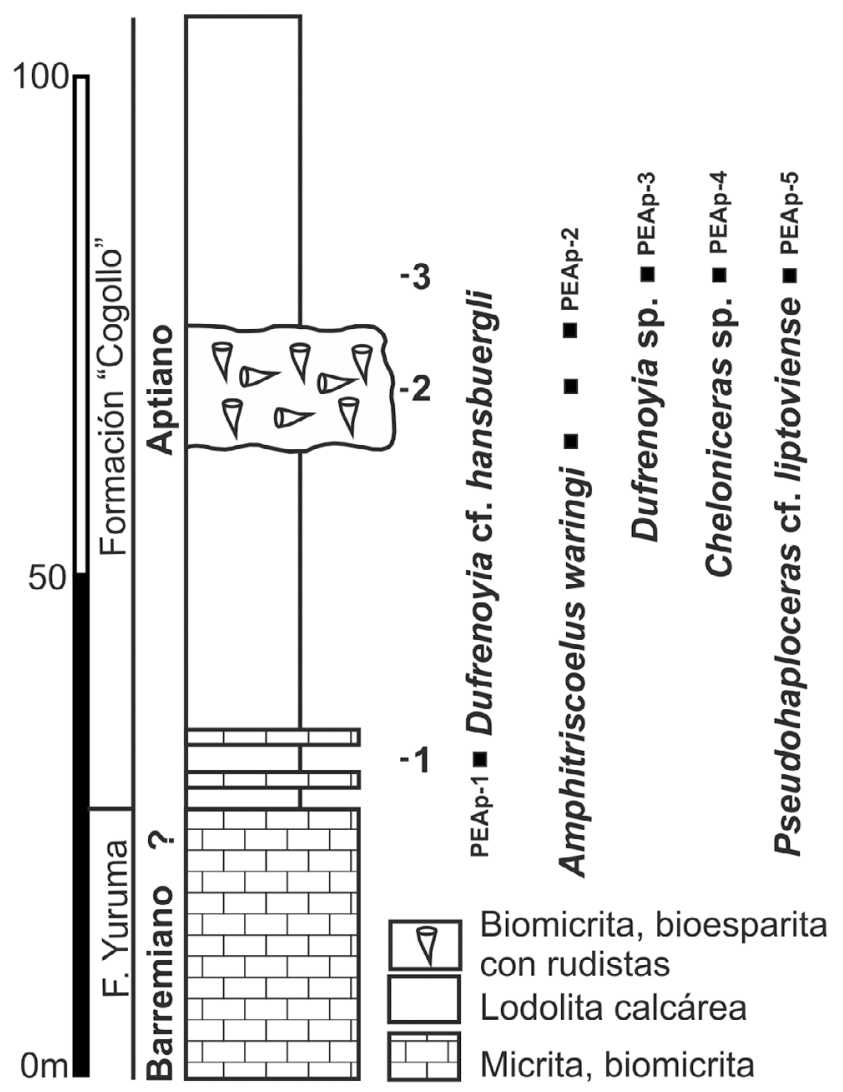

Figura 5. Columna generalizada de la sucesión sedimentaria de los depósitos de la parte alta de la Formación Yuruma y de la parte baja de la Formación "Cogollo" con los puntos de colecta 1, 2 y 3.

\section{Fauna fósil colectada}

Dentro de los depósitos de la parte baja de la Formación "Cogollo" (Figura 5) se identificaron y colectaron macrofósiles bentónicos y nectónicos. El punto de colecta 1 es el más bajo estratigráficamente, el 2 el intermedio y el 3 el más alto (Figura 5).

Si bien para el área de Punta Espada, Renz (1960, p. 335) citó de la parte inferior del Grupo "Cogollo" Cheloniceras, Uhligella, Acantohohoplites y "Gargasites" del Aptiano. En nuestro trabajo de campo, en orden estratigráfico (Figura 5), inicialmente fue posible colectar Dufrenoyia (Figura 1C y Figura 2, punto 1 de colecta), luego estratigráficamente arriba aparece Amphitriscoelus (Figura 1C y Figura 2, punto 2 de colecta) y finalmente encima aparecen Dufrenoyia, Pseudohaploceras y Cheloniceras (Figura 1C y Figura 2, punto 3 de colecta) junto con otros bivalvos.

Dufrenoyia cf. hansbuergli, Etayo-Serna, 1979. PEAp1. Figura 6A-6B. Lugar de colecta 1 en Figura 1C, Figura 5. Se trata de un ejemplar en volumen reemplazado en calcita negra. Las medidas del diámetro (D), altura de la última vuelta (Wh), ancho de la última vuelta $(\mathrm{Ww})$, diámetro umbilical (U) se dan en milímetros y las cifras entre paréntesis son porcentajes del diámetro de la espiral (D). $\mathrm{D}=95,1 ; \mathrm{Wh}=40,5(42,6 \%) ; \mathrm{Ww}=$ 24,8 (26,1\%); $\mathrm{U}=30,8(32,4 \%)$.

La sección de la vuelta es más alta que ancha (Ww/Wh $=61 \%$ ), subelíptica a trapezoidal y más ancha sobre el flanco inferior. La concha es subevoluta y la apertura umbilical es moderada $(\mathrm{U} / \mathrm{D}=32,4 \%)$ siguiendo a Korn (2010). La última vuelta muestra alternativamente costillas principales y secundarias, pero también se pueden reconocer dos principales seguidas. Entre 36 y 37 costillas están presentes en la última vuelta. Las costillas principales con tendencia rectiradiada nacen en la pared umbilical y son suavemente sinuosas. Costillas secundarias inician en el flanco medio. Todas las costillas cruzan el área ventral sin interrupción, aplanadas y ligeramente arqueadas hacia el peristoma, $\mathrm{y}$ en el borde ventrolateral son más gruesas y destacadas. Sobre el flanco y el vientre se reconocen ostras adheridas a la concha, sin poderse afirmar si se adosaron post mortem. 

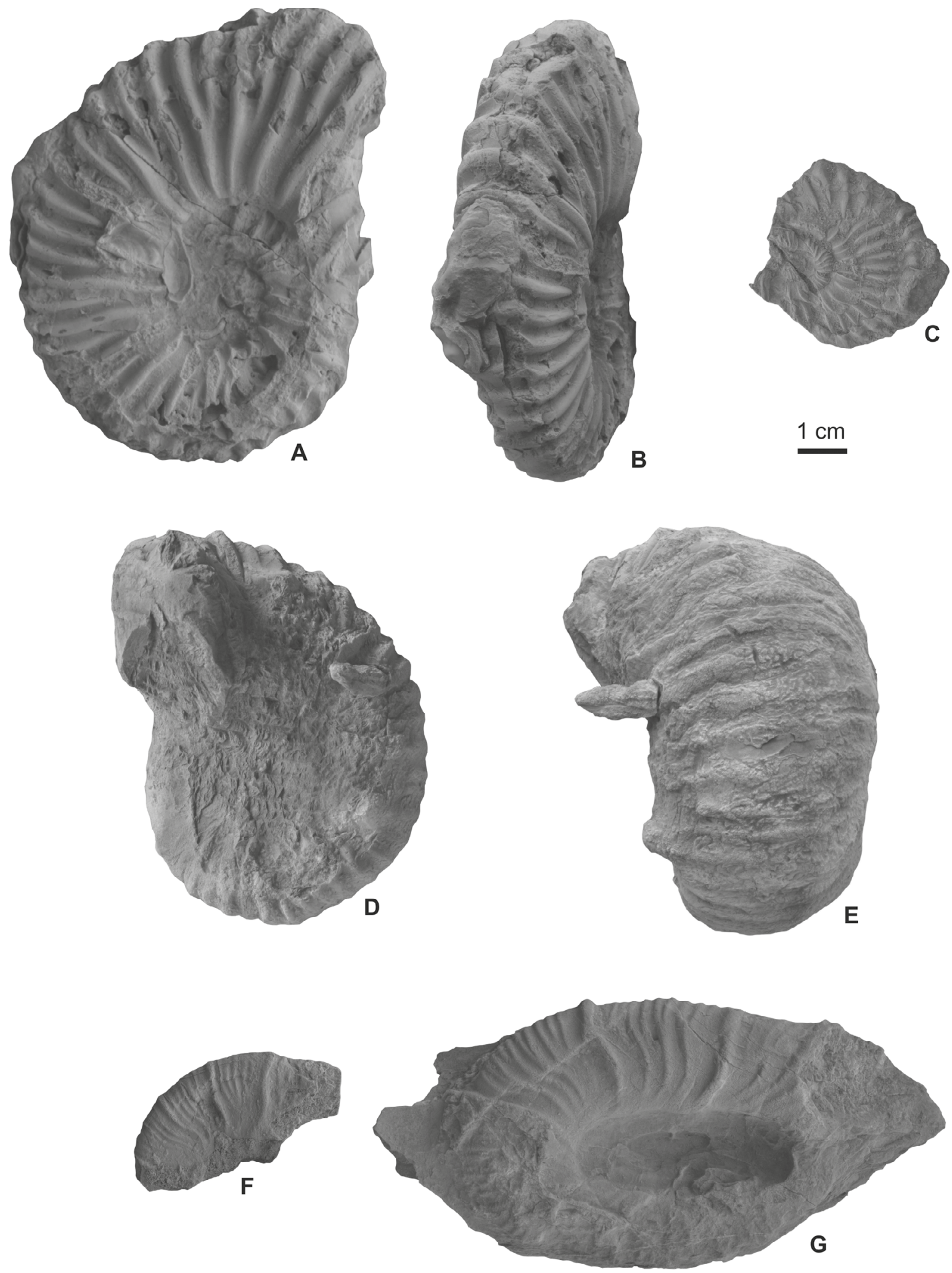

Figura 6. A-B. Dufrenoyia cf. hansbuergli PEAp-1. Lugar 1 de colecta (Figura 1C). C. Dufrenoyia sp. PEAp-3. Lugar 3 de colecta (Figura 1C, Figura 2, Figura 3, Figura 4D, Figura 5). D-E. Cheloniceras sp. PEAp-4. Lugar 3 de colecta (Figura 1C, Figura 2, Figura 3, Figura 4D, Figura 5). F-G. Pseudohaploceras cf. liptoviense PEAp-5. Lugar 3 de colecta (Figura 1C, Figura 2, Figura 3, Figura 4D, Figura 5). 
Etayo-Serna (1979, p. 61, pl. 1, fig. 4) con base en Bürgl (1956) introduce la especie Dufrenoyia hansbuergli dentro de depósitos del Aptiano del sector central de Colombia, que ofrece características similares a las del ejemplar de la Alta Guajira.

Amphitriscoelus waringi Harris y Hodson, 1922. PEAp2. Figura 7A-7E, Figura 8A-8C. Lugar 2 de colecta en Figura 1C, Figura 2, Figura 3, Figura 5. Aparecen conchas prosógiras pequeñas a medianas, inequivalvas con la valva derecha o inferior más larga (Figura 7A, 7B). Tanto en campo como en los cortes realizados se reconocen las tres cavidades principales, los canales paleales simples y los septos no bifurcados (Figura 8). Con base en los cortes y su reconstrucción volumétrica digital se reconoce un contorno subcircular (Figura 8) con superficie externa lisa (Figura 8F-8G).
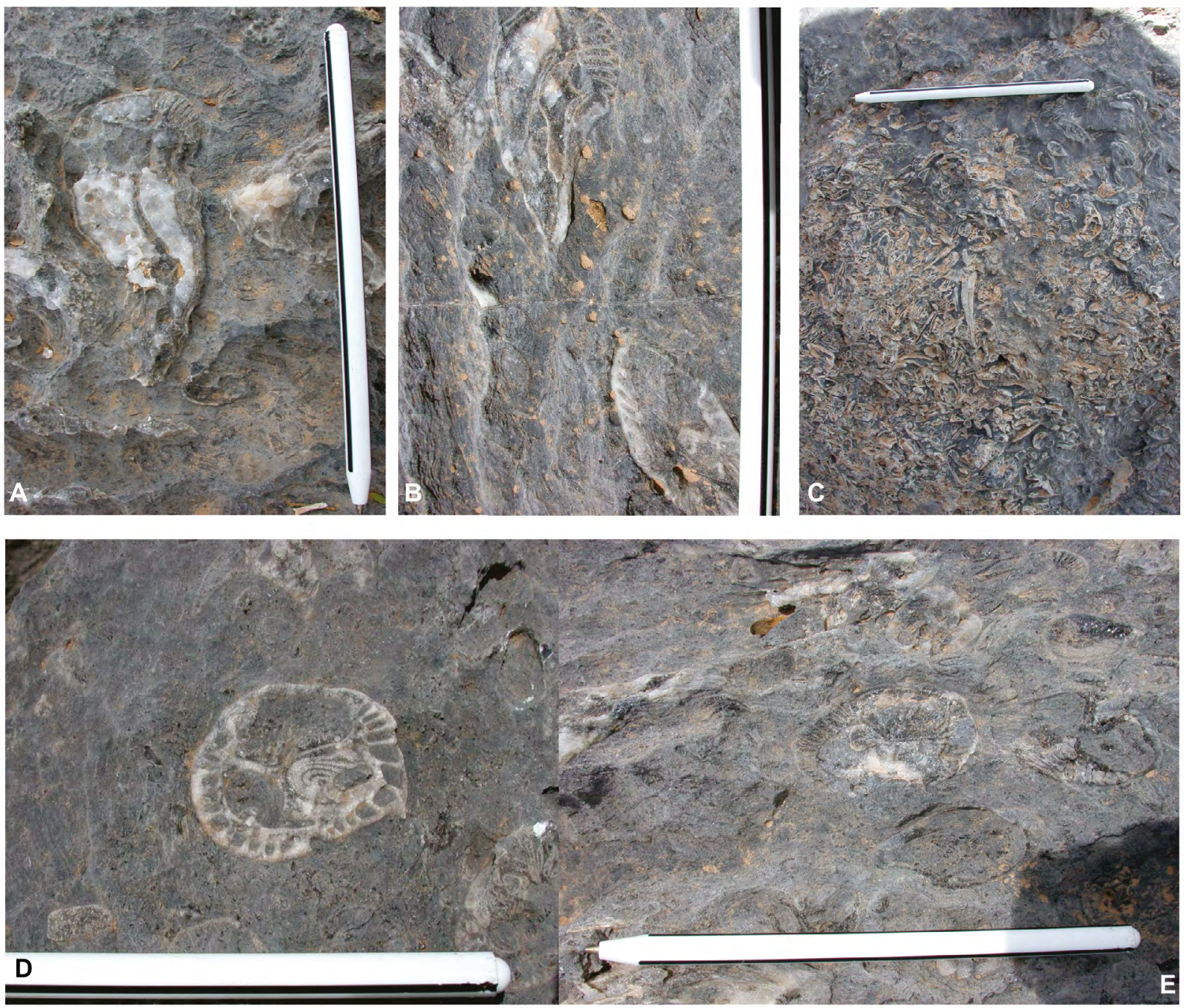

Figura 7. Fotografías de Amphitriscoelus waringi. A-B. Cortes longitudinales de las valvas. C. Concentración de rudistas completos y fragmentados. D-E. Cortes transversales de los rudistas. Lugar 2 de colecta (Figura 1C, Figura 2, Figura 3 ).

Dufrenoyia sp. Figura 6C. PEAp-3. Lugar 3 de colecta en Figura 1C, Figura 2, Figura 3, Figura 5. Ejemplar aplastado con costillas principales y secundarias, en las cuales la sinuosidad y el engrosamiento en el borde ventrolateral es evidente a pesar de la deformación del área ventral.
Cheloniceras sp. Figura 6D-6E. PEAp-4. Lugar 3 de colecta en Figura 1C, Figura2, Figura3, Figura 5. Ejemplar en volumen, reemplazado en calcita, corroído y algo deformado, con sección de la vuelta inflada a deprimida. Las costillas son fuertes con dos hileras de tubérculos claramente reconocidas; una tercera fila no es muy 
clara. Se reconocen espinas sobre el borde ventrolateral (Figura 6D-6E), Ejemplares de "Douvilleiceras Cornueli var. latispinosa" (= Cheloniceras cornuelianum latispinosum, Moreno-Bedmar comunicación verbal) en Nikchitch (1915, pl. 1, fig. 6), al igual que Cheloniceras cornuelianum en Ropolo et al. (2008, pl. 15, fig. 2, 3) muestran tuberculación destacada, algo similar al fósil de Punta Espada.

Pseudohaploceras cf. liptoviense (Zeuschner, 1856). PEAp-5. Figura 6F-6G. Lugar 3 de colecta en Figura
1C, Figura 2, Figura 3, Figura 5. Dos ejemplares deformados en volumen con costillas principales fuertes y costillas secundarias tenues. Todas las costillas son sinuosas. Las costillas principales comienzan en la pared umbilical, las secundarias más largas inician cerca del borde umbilical y las más cortas en el flanco superior. Las costillas secundarias largas pueden bifurcarse. Las constricciones están presentes alrededor de las costillas principales. El ombligo es más o menos abierto.

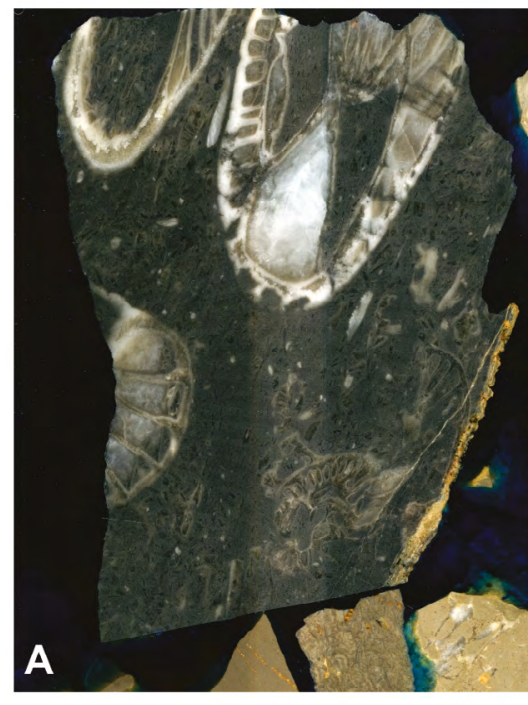

5)

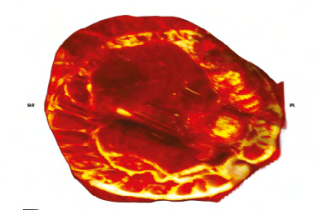

D

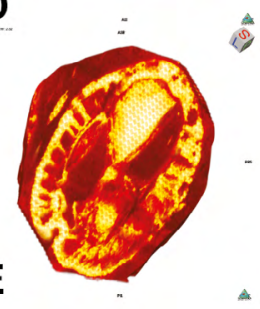

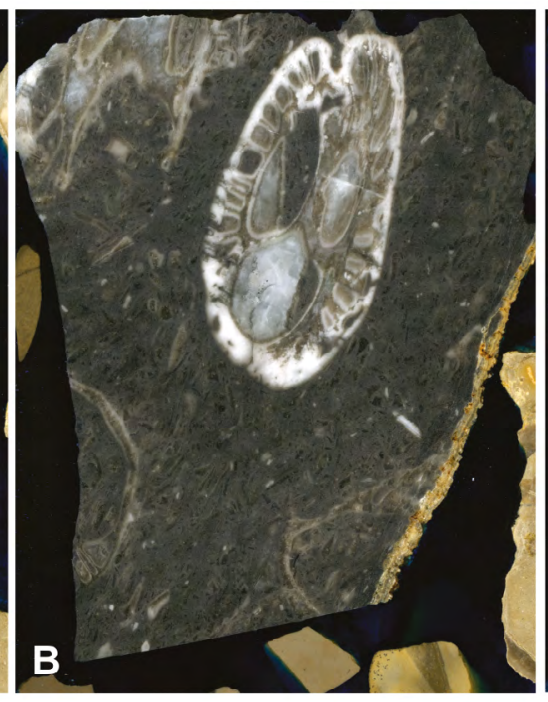

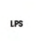
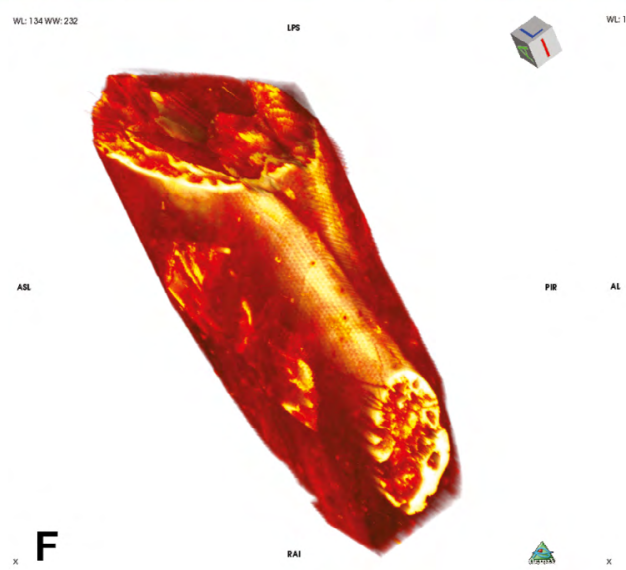
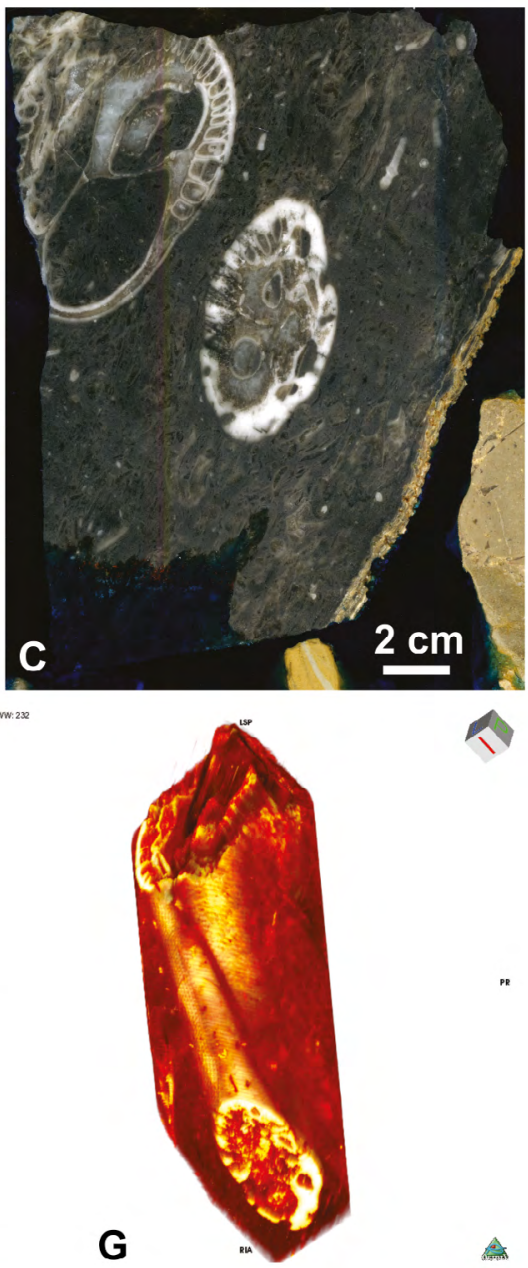

G

离

Figura 8. A-C. Cortes pulidos progresivos de alta resolución realizados por la máquina de corte G\&N MPS 2 - R300 (precision surface grinding). D-G. Reconstrucción digital de Amphitriscoelus waringi con base en los cortes pulidos progresivos de alta resolución realizados por la máquina de corte G\&N MPS 2 - R300 (precision surface grinding). 


\section{Discusión}

\section{Rango Estratigráfico}

Con base en Etayo-Serna (1979), las amonitas del valle Piedra del Destino pueden incluirse en la biozona de asociación de Dufrenoyia sanctorum - Stoyanowiceras treffryanus del Aptiano superior. Pero para Bogdanova y Hoedemaeker (2004) Dufrenoyia es un género del Aptiano inferior y para Ropolo et al. (2008) Cheloniceras es un género del Aptiano inferior.

Sutton (1946, p. 31) reporta en la Isla Toas (Venezuela) A. waringi junto con Cheloniceras sp. y Parahoplites sp. dentro de una sucesión del Aptiano. Además, en depósitos del Aptiano inferior de México $A$. waringi se asocia con Pseudohaploceras liptoviense (Zeuschner) de acuerdo con Alencáster y Pantoja-Alor (1996, p. 400).

Ropolo et al. (2008) indican la presencia de Cheloniceras dentro de la biozona de Deshayesites deshayesi, referida en la biozonación estándar del Mediterráneo por Reboulet et al. (2018) y sobre la que aparece la biozona de Dufrenoyia furcata en la parte más alta del Aptiano inferior. Así que, el rango estratigráfico de los fósiles aquí descritos debe ser considerado del Aptiano inferior.

\section{Consideraciones Paleobiogeográficas}

La influencia de la fauna del mar de Tetis en el norte de Colombia se hace evidente por la presencia de Dufrenoyia, Cheloniceras y Pseudohaploceras. De otro lado, la asociación de Amphitriscoelus con amonitas del Aptiano de la Alta Guajira en Colombia y del norte de Venezuela (von der Osten, 1957; Sutton, 1946; Masse y Rossi, 1987) presentan afinidad con la asociación fósil de México. Dichos rudistas endémicos americanos (Skelton, 1982; Alencáster y Pantoja-Alor, 1996; García-Barrera, 2006, p. 65) o de la provincia Caribe (Kauffman y Johnson, 1988; Patarroyo y Götz, 2014) del Aptiano se reportan en Cuba (Rojas et al., 1992 en Alencáster y Pantoja-Alor, 1996), Jamaica (Brown y Mitchel, 2010), Trinidad (Harris y Hodson, 1922), México (Alencáster y Pantoja-Alor, 1995, 1996, 1998; García-Barrera, 2006), Texas (Harrison y Hodson, 1922), Isla Toas en Venezuela (Sutton, 1946, p. 31), serranía del Interior en Venezuela (von der Osten, 1957; Masse y Rossi, 1987) y en el norte de Colombia (Patarroyo y Götz, 2014).

\section{Consideraciones Tectónicas}

Partiendo de la interacción entre las placas Suramericana y del Caribe, se genera arrastre tectónico sobre los bloques geológicos del área de La Guajira, por lo que se pone en entredicho la continuidad lateral de los depósitos del Cretácico inferior de la serranía del Perijá en dirección a la Alta Guajira. Para comprender el cuestionamiento, se debe tener en cuenta entonces la paleogeografía y la paleoposición de los bloques ubicados al norte de las fallas de Oca y de Cuisa, considerando además diferentes modelos tectónicos como los de Hernández et al. (2003) y Ramírez et al. (2015, fig. 33), que ubican dichos bloques al oeste, en donde las condiciones sedimentológicas y ambientales eran muy diferentes a las del sur en la cuenca central del Cretácico colombiano y en la serranía del Perijá en Colombia y en Venezuela.

Dichas condiciones sedimentológicas y paleoecológicas pueden indicar también una paleoposición muy diferente de la zona de depósito en relación con su actual cercanía al sector de la serranía del Perijá.

\section{Conclusiones}

La historia geológica de la Alta Guajira debe ser estudiada sin prejuicios para obtener una mejor comprensión de su evolución geológica y en consecuencia de sus unidades litoestratigráficas.

Parte de la nomenclatura litoestratigráfica de la sucesión del Cretácico de la Alta Guajira es traída de la geología de sectores aledaños, los cuales no tienen relación geológica directa entre sí.

En la Alta Guajira se usa el término Cogollo de la nomenclatura litoestratigráfica de la zona de la serranía del Perijá en Colombia y Venezuela. Si bien la unidad litoestratigráfica reconocida como Cogollo en ambos sectores, presenta algunas características litológicas similares, no es recomendable que se continué empleando el término Cogollo en la Alta Guajira, más aún cuando las unidades litoestratigráficas infrayacentes poseen denominaciones diferentes y con historias de depósito igualmente diferentes.

Los depósitos del Aptiano en el sector de Punta Espada están representados por biomicritas, bioesparitas y lodolitas calcáreas que incluyen fósiles del Aptiano. 
Dentro de estos depósitos del valle Piedra del Destino (Punta Espada, Uribia - La Guajira, Colombia) se hallaron Dufrenoyia sp., D. cf. hansbuergli, Cheloniceras sp. Pseudohaploceras cf. liptoviense $\mathrm{y}$ Amphitriscoelus waringi que representan un rango estratigráfico del Aptiano inferior.

Si bien existen fósiles del dominio del mar del Tetis, el reporte de $A$. waringi, que está presente en México, Texas, Venezuela y en islas del Caribe, indica cercanía y afinidad paleogeográfica por su provincialismo.

\section{Agradecimientos}

Nuestros más sinceros agradecimientos a la comunidad de Punta Espada. Además, a Edwar Salazar y a los estudiantes de la práctica de campo (2009) del Departamento de Geociencias, Universidad Nacional de Colombia por todo el apoyo proporcionado.

A los evaluadores, doctores Javier Aguilar Pérez (Facultad de Ciencias de la Tierra, Universidad Autónoma de Nuevo León, México) y Josep A. Moreno-Bedmar (Instituto de Geología, Universidad Nacional Autónoma de México, México), un especial agradecimiento por los valiosos comentarios y opiniones, ya que contribuyeron a mejorar la presente contribución.

P.P. igualmente agradece al Servicio Alemán de Intercambio Académico (DAAD, becas A/03/06854 y A/06/0736), y al convenio ALECOL-DAAD, Ministerio de Educación Nacional de Colombia, Colciencias ICETEX (beca A/09/74196) por la financiación del trabajo de investigación en la Universidad de Heidelberg (Alemania).

\section{Referencias}

Alencáster, G.; Pantoja-Alor, J. (1995). New early Aptian rudist (Bivalvia-Hippuritacea) from Huetamo area in Southwestern Mexico. Revista Mexicana de Ciencias Geológicas, 12(2), 123134.

Alencáster, G.; Pantoja-Alor, J. (1996). The rudist Amphitriscoelus (Bivalvia-Hippuritacea) in the lower Cretaceous of Southwestern Mexico. Journal of Paleontology, 70(3), 399-407. https:// doi.org/10.1017/S0022336000038336
Alencáster, G.; Pantoja-Alor, J. (1998). Two new Lower Cretaceous rudists (Bivalvia-Hippuritacea) from the Huetamo Region; Southwestern Mexico. Geobios, M.S. 22, 15-28. https://doi.org/10.1016/ S0016-6995(98)80061-2

Álvarez, W. (1967). Geology of the Simarua and Carpintero areas, Guajira Peninsula, Colombia. Ph.D. Thesis, Princeton University, USA.

Bogdanova, T.N.; Hoedemaeker, Ph.J. (2004). Barremian-Early Albian Deshayesitidae, Oppeliidae, Desmoceratidae and Silesitidae of Colombia. Scripta Geologica, 128, 183-312.

Brown, I.; Mitchel, S.F. (2010). Lithostratigraphy of the Cretaceous succession in the Benbow Inlier, Jamaica. Caribbean Journal of Earth Science, 41, 25-37.

Bürgl, H. (1956). La variabilidad de la amonita Dufrenoyia texana Burckhard. Boletín Geológico, 4(2-3), 1-22.

Bürgl, H. (1960). Geología de la Península de la Guajira. Boletín Geológico, 6(1-3), 129-168.

Cáceres, H.; Camacho, R.; Reyes, J. (1980). The Geology of the Rancheria Basin. Colombian Society of Petroleum Geologists and Geophysicists. Geological Field - Trip Colombia, 1980 - 1989. Publicado en Colombia por Geotec Ltda. 1992.

Durham, J.W. (1946). Stratigraphy of the CesarRancheria Valleys and the Guajira Peninsula. Geological Report N. 539, Tropical Oil Company.

Etayo-Serna, F. (1979). Zonation of the Cretaceous of central Colombia by ammonites. Publicaciones Geológicas Especiales del Ingeominas, 2, 1-186.

Etayo-Serna, F. (1981). On the suppossed Aptian occurrence of the ammonite genus Neodeshayesites in Colombia and Venezuela; with an appendix on Neodeshayesites karsteni (Marcou). Geología Norandina, 3, 45-51.

García-Barrera, P. (2006). Rudists of Mexico: Historical Aspects and Selected Areas of Research. En: F.J. Vega; T.G. Nyborg; M. Perrilliat; M. MontellanoBallesteros; S.R.S. Cevallos-Ferriz; S.A. Quiroz- 
Barroso (eds.). Studies on Mexican Paleontology (pp. 61-78). Springer.

Garner, A. (1926). Suggested nomenclature and correlation of geological formations in Venezuela. Transactions of the American Institute of Mining and Metallurgical Engineers, 677-684.

Gómez, I. (2001). Structural style and evolution of the Cuisa Fault System, Guajira, Colombia. MSc. Thesis, University of Houston, USA.

Govea, C.; Dueñas, E. (1975). Informe geológico preliminar de la Cuenca del Cesar. Ecopetrol. Informe 390.

Harris, G.D.; Hodson, F. (1922). The rudists of Trinidad. Paleontographica Americana, 1, 119162.

Hernández, M. (2003). Memoria explicativa geología plancha 48, Jagua de Ibirico. Escala 1: 100.000, INGEOMINAS.

Hernández, R.; Ramírez, V.; Reyes, J. (2003). Evolución Geohistórica de las Cuencas del Norte de Colombia. VIII Simposio Bolivariano - Exploración Petrolera en las Cuencas Subandinas. Cartagena, Colombia.

Irving, E.M. (1971). La evolución estructural de los Andes más septentrionales de Colombia. Boletín Geológico, 19(2), 1-89.

Kauffman, E.G.; Johnson, C. (1988). The morphological and ecological evolution of Middle and Upper Cretaceous reef-building rudistids. Palaios, 3(2), 194-216. https://doi.org/10.2307/3514530

Korn, D. (2010). A key for the description of Palaeozoic ammonoids. Fossil Record, 13(1), 5-12. https:// doi.org/10.1002/mmng.200900008

MacDonald, W.D. (1964). Geology of the Serranía de Macuira Area, Guajira Peninsula, Colombia. Ph.D. Thesis, Princeton University, USA.

MacDonald, W.D. (1965). Geology of the Serrania de Macuira area Gujira peninsula, Northeast Colombia. En: J.B. Saunders (ed.). Transactions of the Fourth Caribbean Geological Conference (pp. 267-274). Caribbean Printers.
Masse, J.P.; Rossi, T. (1987). Le provincialisme sudcaraibe à l'Aptien inférieur. Sa signification dans le cadre de l'evolution geodynamique du domaine Caraibe et de l'Atlantique central. Cretaceous Research, 8(4), 349-363. https://doi. org/10.1016/0195-6671(87)90004-8

Mercado, M. (1999). Geología del departamento de La Guajira. Escala 1:250.000. INGEOMINAS.

Nikchitch, J. (1915). Représentants du genre Douvilleiceras de l'Aptien $d u$ versant septentrional du Caucase. Memoires du Comité Geologique, nouvelle serie, libraison 121. Paris, Francia. Librairie scientifique A. Hermann.

Notestein, F.B.; Hubman, C.W.; Bowler, J.W. (1944). Geology of the Barco Concession, Republic of Colombia, South America. GSA Bulletin, 55(10), 1165-1216. https://doi.org/10.1130/GSAB-551165

Patarroyo, P. (2011). Observaciones sobre la geología de las sedimentitas del Cretácico (BarremianoConiaciano) del sector de Punta Espada, Alta Guajira (Uribia-Guajira-Colombia). XIV Congreso Latinoamericano de Geología y XIII Congreso Colombiano de Geología. Medellín, Colombia.

Patarroyo, P. (2020). Barremian deposits of Colombia: A special emphasis on marine successions. En: J. Gómez; A.O. Pinilla-Pachón (eds.). The Geology of Colombia (pp. 445-474). Volume 2, Chapter 12. Servicio Geológico Colombiano. https://doi. org/10.32685/pub.esp.36.2019.12

Patarroyo, P.; Götz, S. (2013). Dufrenoyia y Amphitriscoelus en depósitos del Aptiano de la Formación Cogollo (Guajira - Colombia). XIV Congreso Colombiano de Geología, Bogotá, Colombia.

Patarroyo, P.; Götz, S. (2014). Aptian ammonite and rudist association, Cogollo Formation (Guajira Colombia). Paleobiogeographic significance to north Colombia. 23rd Latin American Colloquium on Earth Sciences. Heidelberg, Germany.

Ramírez, V.; Vargas, L.E.; Rubio, C.; Niño, H.; Mantilla, O. (2015) Petroleum systems of the Guajira Basin, northern Colombia. En: C. Bartolini; P. Mann (eds.). Petroleum geology and 
potential of the Colombian Caribbean Margin (pp. 399-430). Memoir 108. AAPG. https://doi. org/10.1306/13531944M1083647

Reboulet, S.; Szives, O.; Aguirre-Urreta, M.B.; Barragán, R.; Company, M.; Frau, C.; Kakabadze, M.; Klein, J.; Moreno-Bedmar, J.A.; Lukeneder, A.; Pictet, A.; Ploch, I.; Raisossadat, S.; Vašíček, Z.; Baraboshkin, E.J.; Mitta, V. (2018). Report on the 6th International Meeting of the IUGS Lower Cretaceous Ammonite Working Group, the Kilian Group (Vienna, Austria, 20th August 2017). Cretaceous Research, 91, 100-110. https://doi. org/10.1016/j.cretres.2018.05.008

Renz, O. (1956). Cretaceous in Western Venezuela and the Guajira (Colombia). XX Congreso Geológico Internacional, Ciudad de México, México.

Renz, O. (1959). Estratigrafía del Cretáceo de Venezuela occidental. Boletín de Geología (Caracas), 5(10), 3-48.

Renz, O. (1960). Geología de la parte sureste de la Peninsula de la Guajira (República de Colombia). Mem. 1161, Congreso Geológico Venezolano, 1, Minist. Min. Hidrocarburos, Publicación Especial, 3, 317-347.

Richards, H.G. (1968). Cretaceous section in Barco area of northeastern Colombia. AAPG Bulletin, 52(12), 2324-2336. https://doi.org/10.1306/5D25C57F16C1-11D7-8645000102C1865D

Rod, E.; Maync, W. (1954). Revision of Lower Cretaceous stratigraphy of Venezuela. AAPG Bulletin, 38(2), 193-283. https:// doi.org/10.1306/5CEADECC-16BB-11D7$8645000102 \mathrm{C} 1865 \mathrm{D}$

Rollins, J.F. (1960). Stratigraphy and structure of the Goajira Peninsula, Northweastern Venezuela and Northeastern Colombia. Ph.D. Thesis, University of Nebraska, USA.

Rollins, J.F. (1965). Stratigraphy and structure of the Goajira Peninsula, Northwestern Venezuela and Northeastern Colombia. University of Nebraska Studies, 30, 1-102.
Ropolo, P.; Conte, G.; Moullade, M.; Tronchetti, G.; Gonnet, R. (2008). The Douvilleiceratidae (Ammonoidea) of the Lower Aptian historical stratotype area at Cassis-La Bédoule (SE France). Carnets de Géologie / Notebooks on Geology, Brest, Memoir 2008/03, 1-60.

Salazar, E. (2010). Análisis estratigráfico y determinación de ambientes de depósito para la Formación Palanz. Inicio de la sedimentación cretácica en la Alta Guajira, Colombia. Tesis de Maestría, Universidad Nacional de Colombia, Bogotá, Colombia.

Skelton, P. (1982). Aptian and Barremian rudist bivalves of the New World: Some Old World similarities. Cretaceous Research, 3(1-2), 145-153. https:// doi.org/10.1016/0195-6671(82)90015-5

Sutton, F. (1946). Geology of the Maracaibo basin, Venezuela. AAPG Bulletin, 30(10), 1621-1741.

von der Osten, E. (1957). A Fauna from the Lower Cretaceous Barranquín Formation of Venezuela. Journal of Paleontology, 31(3), 571-590.

Zeuschner, L. (1856). Geognostische Beschreibung des Liaskalkes in der Tatra und in den angrenzenden Gebirgen. Sitzungsberichte der kaiserlichen Akademie der Wissenschaften. MathematischNaturwissenschaftliche Classe. Neunzehnter Band, Heft I und II, 135-182.

Zuluaga, C.; Ochoa, A.; Muñoz, C.; Guerrero, N.; Martínez, A.; Medina, P.; Pinilla, A.; Ríos, P.; Rodríguez, P.; Salazar, E.; Zapata, V. (2009). Memoria de las planchas 2, 3, 5, y 6 (con parte de las planchas 4, 10 y 10bis). Proyecto de investigación: Cartografía e Historia Geológica de la Alta Guajira, implicaciones en la búsqueda de recursos minerales. Universidad Nacional de Colombia - INGEOMINAS.

Trabajo recibido: mayo 25 de 2020

Trabajo aceptado: agosto 19 de 2020 